\title{
¿Las cercas vivas ayudan a la conservación de la diversidad de mariposas en paisajes agropecuarios?
}

\author{
Diego Enrique Tobar L. ${ }^{1}$ \& Muhammad Ibrahim ${ }^{1}$ \\ 1. Programa de Ganadería y Manejo del Medio Ambiente (GAMMA), Centro Agronómico Tropical de Investigación y \\ Enseñanza (CATIE), A.A. 7170, Turrialba, Costa Rica; dtobar@catie.ac.cr
}

Recibido 13-III-2009. Corregido 10-VI-2009. Aceptado 14-VII-2009.

\begin{abstract}
Do live fences help conserve butterfly diversity in agricultural landscapes? In Central America, natural forests have been transformed into agriculture production areas, generating forest fragmentation, desertification, erosion and loss of biodiversity, among other concerns. Different tree cover compositions are kept on these agricultural landscapes, including scattered trees in pastures, live fences, fragments of secondary forests, and riparian forests. These can help in biodiversity conservation because they generate shelter, feeding and reproduction areas, among others. We studied the composition, richness and abundance of diurnal butterflies on two types of live fences in a landscape where pastures are predominant in Costa Rica's Central Pacific Region. Transects $(120 \times 5 \mathrm{~m})$ were observed for an hour (two days/habitat) in five multi-strata fences (with several plant species, strata and canopy width) and five simple fences (with smaller and pruned trees). A total of 2782 butterflies were observed (75 species). The most abundant species were Anartia fatima, Eurema daria, Eurema nise, Hermeuptychia hermes, Junonia evarete and Phoebis philea. Multi-strata fences had more species and can help maintain $56 \%$ of the total species observed in secondary and riparian forests. This type of live fence can play an important role in butterfly conservation in livestock areas, and its benefits are influenced by the manner in which farmers manage their land. Rev. Biol. Trop. 58 (1): 447-463. Epub 2010 March 01.
\end{abstract}

Key words: Biodiversity, Costa Rica, environmental services, fragmentation, habitat, guild, live fences, Papilionoidea, silvopastoral systems, richness of species.

La fragmentación es el proceso de la destrucción parcial de un hábitat natural que da como resultado la producción de fragmentos de bosque de diferentes tamaño, forma y grado de aislamiento (Murcia 1995) e interrupción de la conectividad (Bennet 1999). Esto es una de las principales amenazas para la conservación de la diversidad y para la generación de servicios ambientales (Zhang et al. 2001, Balmford \& Bond 2005).

En Centroamérica muchos paisajes han sido fragmentados, deforestados y transformados en áreas agropecuarias, lo que ha generado mosaicos de pequeños relictos de bosque inmersos en una matriz de pasturas. La FAO (2003) ha estimado que el $40 \%$ de la totalidad del territorio Centroamericano está constituido por tierras utilizadas para pastoreo. A su vez, un $60 \%$ de estas áreas de pasturas se encuentran degradadas, como consecuencia de la producción ganadera tradicional basada en el manejo de pasturas sin árboles. Esto ha ocasionado diferentes problemas ambientales, como la erosión y pérdida de la fertilización natural de los suelos, desertificación, pérdida de diversidad biológica, emisión de gases de efecto invernadero y contaminación de aguas. Asimismo, ha influido en el cambio de la composición de las especies de las comunidades bióticas, alteración en la función de los ecosistemas, propiciando la dispersión de especies exóticas y las que se benefician de la 
fragmentación del hábitat (Kaimowitz 2001, Harvey et al. 2008).

Actualmente, se ha planteado que una estrategia para solventar el impacto ambiental y la productividad de los sistemas convencionales de producción, es el uso de los sistemas silvopastoriles (SSP), que ayudan a incrementar la sostenibilidad y productividad de las fincas ganaderas (Devendra \& Ibrahim 2004; Kallenbach et al. 2006). Un SSP es una estrategia de producción pecuaria que involucra la presencia de especies leñosas perennes (árboles o arbustos) y de los componentes tradicionales (forrajeras herbáceas y animales), en donde todos ellos interactúan bajo un sistema de manejo integral (Somarriba 1992). Algunos sistemas silvopastoriles son: leñosas en callejones, pastoreo en plantaciones de árboles maderables o frutales, barreras vivas y cortinas rompevientos, cercas vivas, árboles y arbustos dispersos en potrero y bancos forrajeros (Pezo \& Ibrahim 1998). Estos sistemas son una alternativa para incrementar o mantener la producción pecuaria y el establecimiento o manejo de la cobertura arbórea en fincas ganaderas para de la generación de servicios ambientales (Ibrahim et al. 2000), de la misma manera se espera poder realizar un manejo sostenible de los recursos naturales.

Las cercas vivas y los árboles dispersos en potreros, al igual que los sistemas silvopastoriles y agroforestales en general, son hábitats perturbados y manejados por el hombre que han demostrado tener un papel importante en la conservación de los suelos, clima, agua y diversidad biológica (Casasola et al. 2009). Diversos estudios han mencionado que las cercas vivas y los árboles dispersos en potreros pueden contribuir a la conservación de la biodiversidad, debido a que pueden servir como corredores biológicos para la fauna y flora silvestre (Beer et al. 2003), incrementan la conectividad estructural de los paisajes, fomentan la cobertura arbórea en áreas de pasturas y permiten que estás áreas sean menos contrastantes con los fragmentos de bosque (Chacón \& Harvey 2008), también proveen servicios ambientales como almacenamiento de carbono, conservación de la biodiversidad (Guevara et al. 1998; 2005, Estrada \& Coates-Estrada 2002, 2005; Pagiola et al. 2004; Harvey et al. 2005) y protección de fuentes de agua (Ríos et al. 2007).

Estudios recientes han evidenciado que paisajes fragmentados o agropecuarios pueden mantener una proporción de la biodiversidad original al exhibir una cobertura arbórea en forma de relictos de bosque, charrales o tacotales, cercas vivas y árboles dispersos (Daily et al. 2001, Cárdenas et al. 2003, Lang et al. 2003, Petit \& Petit 2003, Harvey et al. 2006, Sáenz et al. 2007, Tobar et al. 2007). Las cercas vivas son elementos lineales que dependiendo de la estructura vertical o el número de estratos pueden generar hábitats, dependiendo de la abundancia y composición florística, estructura arbórea y arreglo espacial de las cercas en el agropaisaje, resultan de gran importancia para la conservación (Harvey et al. 2005). Es decir que las cercas vivas que presentan una mayor complejidad estructural y mayor diversidad florística, pueden ayudar a conservar un mayor número de especies de fauna silvestres que las cercas vivas simples (Lang et al. 2003, Harvey \& Haber 1999; Harvey et al. 2008). Sin embargo, no sabemos qué especies de mariposas pueden estar utilizando este tipo de hábitats lineales y cuáles de ellas los utilizan para sus movimientos en los agropaisajes.

Para la realización de dichos estudios se ha establecido el uso de especies indicadoras, cuyos criterios de selección se basan en: (1) la diversidad y conocimiento del grupo taxonómico, (2) ser de fácil observación, (3) una historia natural bien conocida, (4) la sensibilidad a los cambios en su hábitat natural, y (5) responder de manera rápida a los cambios ambientales (McGeoh et al. 2002). Las mariposas son un grupo altamente sensible a los cambios ambientales ocasionados por la alteración y la fragmentación del hábitat, así como al cambio climático. Por lo anterior, el uso de los lepidópteros ha resultado muy atractivo para estudios de ecología, biogeografía, programas de monitoreo en áreas bajo manejo forestal, y podrían jugar un papel importante, para evidenciar el 
valor de conservación de los diferentes usos de la tierra en paisajes agropecuarios, como es el uso de las cercas vivas simples y multiestrato, empleadas en las fincas ganaderas para dividir potreros o marcar los límites o linderos de las fincas. El estudio tuvo como propósito evaluar la abundancia, diversidad y riqueza de especies de mariposas presentes en las cercas vivas simples y multi-estratos en un paisaje agropecuario en la región del Pacifico Central de Costa Rica

\section{MATERIALES Y MÉTODOS}

Área de estudio: Se encuentra localizada entre los Distritos de Montes de Oro, Esparza, Puntarenas, provincia de Puntarenas y San Ramón, Alajuela (0958'60" N y $\left.84^{\circ} 40^{\prime} 00^{\prime \prime} \mathrm{W}\right)$, perteneciente al la región Pacífica Central de Costa Rica. La temperatura media anual es de $27^{\circ} \mathrm{C}$, precipitación anual entre 1500 y $2000 \mathrm{~mm}$, con una altitud entre 300 y $1000 \mathrm{msnm}$. La época lluviosa dura siete meses (mayo-noviembre) y cinco meses la época seca (diciembre-abril). La humedad relativa está entre 65 a $80 \%$. La topografía presenta pendientes que varían entre 0 y $30 \%$. La vegetación predominante pertenecen a la zona de vida de bosque subhúmedo tropical (Holdridge 1963).

En Esparza, el uso de cercas vivas y árboles dispersos en potreros, son prácticas comunes, en donde los árboles que se utilizan provienen de: la regeneración natural el $70 \%$, $15 \%$ son árboles remanentes y $15 \%$ han sido plantados. La actividad predominante es la producción pecuaria, el $63 \%$ de las fincas tienen un sistema de producción de carne (cría y/o engorde); el 34\% son fincas con un sistema de producción bovina de doble propósito (leche y carne); y un 3\% comprende fincas con sistemas de producción diversos (leche, agricultura+ganadería y agricultura). Existen diversas razas de ganado bovino: en fincas con ganado para carne predominan Brahman, cruces de Brahman con Indo brasil; en fincas con ganado de doble propósito sobresalen animales cruzados como cebú (Brahman o Brahman con Indo brasil), con razas lecheras (Holstein o pardo suizo) (Villanueva et al. 2007).
Las especies de pastos más utilizadas son Brachiaria brizantha e Hyparrhenia rufa. El paisaje se encuentra conformado por una matriz predominante de pasturas, remanentes de bosque, cultivos de caña y plantaciones forestales $(66,20,10,4 \%$, respectivamente). Los bosques ribereños son de escasa extensión y de formas lineales, que en su mayoría no superan los $50 \mathrm{~m}$ de ancho. La red de cercas vivas se encuentra dominada por Bursera simaruba (Indio pelado) y Bombacopsis quinata (Pochote). Las cercas vivas dominadas por estas especies, son podadas cada año entre $2-3 \mathrm{~m}$ de altura. Las fincas presentan diferentes tipos de cobertura arbórea, las cuales son conservadas por los productores para múltiples propósitos (leña, madera, sombra para ganado, entre otros; Villanueva et al. 2007).

Selección de las cercas vivas: Se seleccionaron 10 cercas vivas, que tuvieran dos tipos de estructuras: (1) cinco cercas vivas simples (CVS): compuestas por Bursera simaruba (Indio pelado) o Bombacopsis quinata (Pochote) y en algunos casos, una combinación de otras especies. Estas cercas presentaron postes con diámetro a la altura del pecho (dap) menores a $10 \mathrm{~cm}$, un ancho promedio de copa menor de $4 \mathrm{~m}$ y una altura promedio menor de $6 \mathrm{~m}$. (2) cinco cercas vivas multiestrato o complejas (CVM): conformadas por más de dos especies arbóreas y arbustivas de diferentes diámetros, alturas y ancho de copa mayor a $6 \mathrm{~m}$, de aproximadamente $200 \mathrm{~m}$ de longitud, localizadas en potreros de las fincas ganaderas.

Observaciones de mariposas: El muestreo de mariposas se realizó durante el año 2005 y los meses de febrero a mayo de 2006 . Cada cerca fue visitada cuatro veces durante la época seca y cuatro veces durante la época de lluvia, los transectos fueron recorridos a lo largo del día por un lapso de 45 minutos entre las 8:00 y las 16:00 horas. Se recorrió a un paso constante y uniforme, registrando, contando los individuos adultos de cada especie de mariposa, dentro de un margen de $2.5 \mathrm{~m}$ a cada lado de la línea base del transecto. Para un total de 
3h por cerca viva/periodo de muestreo, y $90 \mathrm{~h}$ totales de esfuerzo de muestreo.

Las capturas de las especies de mariposas se efectuaron mediante una red entomológica, y se registró los siguientes datos: número de individuos, número del transecto, tipo de cerca viva, altitud, actividad (percha, cópula, alimentación, entre otras) y la hora. Los individuos pertenecientes a especies difícilmente reconocibles al vuelo o desconocidas fueron recolectados para su identificación mediante claves e ilustraciones de revisiones taxonómicas (D'Abrera 1981, 1984, 1987a, 1987b, 1989, 1994, 1995, DeVries 1987, 1997, Lamas 2004, Chacón \& Montero 2007).

Caracterización vegetal: En cada cerca viva utilizada para la observación de mariposas, se estableció una parcela lineal de $200 \mathrm{~m}$, donde se realizó un inventario completo de todos los árboles con un diámetro a la altura del pecho (dap) mayor o igual a $5 \mathrm{~cm}$. Cada individuo se identificó a especie, se tomaron las variables dasométricas como altura total, altura del fuste o comercial, dap y diámetro de copa. Las plantas no identificadas fueron llevadas al Herbario Juvenal Rodríguez de la Universidad Nacional para su posterior identificación. Los arbustos se midieron caminando a lo largo de las cuerdas con los brazos extendidos $(1.5 \mathrm{~m})$ desde el centro hacia los puntos cardinales y contando los individuos con diámetro menor a $3 \mathrm{~cm}$ que fueron tocados por los brazos o el cuerpo al nivel del pecho. Los arbustos fueron considerados dentro de la primera categoría de dap.

Para cada tipo de cerca viva (simple y multiestrato), se calculó la abundancia, la riqueza de mariposas y los índices de diversidad de Shannon, Simpson (Magurran 2003). Estos resultados fueron comparados estadísticamente mediante una prueba $\mathrm{T}$ unilateral para dos muestras, para probar la hipótesis que las cercas vivas multiestrato se mantienen más diversas que las simples. Los análisis fueron realizados mediante el programa estadístico InfoStat V.2007p.
Se realizaron curvas de acumulación de especies mediante el reordenamiento aleatorio repetido con 1000 aleatorizaciones de los datos con el programa EstimateS (Colwell 2004). Para evaluar el nivel de inventario en cada cerca se usaron dos estimadores: el estimador de segundo orden de Chao (Colwell \& Coddington 1994) y el modelo de Clench (Soberon \& Llorente 1994). La diversidad entre hábitats de la comunidad de mariposa se estimó mediante el índice de complementariedad (Colwell \& Coddington 1994), el cual expresa la diferencia, en porcentaje, de la composición en las listas de especies de dos hábitats. El valor mínimo de complementariedad (C) es cero cuando las listas de especies de los dos hábitats son idénticas y el valor máximo de 100 indica que las listas son completamente diferentes.

Para detectar si la proporción de gremios alimenticios de mariposas (nectarívoros: se alimentan de polen y néctar de las flores; generalistas: se alimenta de frutos, savias, excretas entre otras) (DeVries 1987); las especies fueron agrupadas bajo estas dos categorías, las cuales fueron comparadas mediante una prueba de bondad de ajuste $\mathrm{G}$ para un cuadro de contingencia de $2 \times 2$, el cual fue realizado con el programa Infostat V. 2007p.

Para la selección de variables de hábitat (cercas vivas simples y multiestrato), se usó un análisis de gradiente para determinar la variación de la riqueza de especies de mariposas a lo largo del gradiente de los tipos de cerca seleccionadas. Primero, se realizó un análisis de correspondencia rectificado (Detrended Correspondence Analysis, DCA) con variables previamente estandarizadas; el mayor valor del primer Eje X permitió elegir el tipo de análisis siguiente, lineal (longitud del eje $<3$ ) y unimodal (longitud del eje $>4$ ) para valores intermedios.

Se realizó un análisis de correspondencia canónico (CCA, Ter Braak \& Smilauer 2002) cuando la respuesta de las variables fue unimodal; y cuando las variables tuvieron una respuesta lineal, se usó un análisis de redundancia (RDA). Posteriormente, se seleccionó la variable que mejor explicó la riqueza de 
mariposas usando un modelo de regresión paso a paso (stepwise forward) y probando la significancia de la selección mediante permutaciones de Montecarlo con pruebas de F. El criterio para mantener una variable dentro el modelo fue de $\mathrm{p}<0.05$ y para retirarla $\mathrm{p}>0.10$. Con las variables seleccionadas (regresoras), se realizó un ajuste usando un modelo lineal general (GLM) y el número de especies de mariposas como variable dependiente; de esta manera, se determinó en algunos casos el óptimo de esta variable, el cual a su vez produjo un óptimo de riqueza de mariposas. Para el mejor ajuste del modelo, se usó el criterio de Akaike (AIC, Akaike Information Criterion) y valores de F. La mejor variable fue la que tuvo el menor valor de AIC y un valor F significativo. En los análisis se emplearon los programas CANOCO 4.5 para Windows (Ter Braak \& Smilauer 2002).

\section{RESULTADOS}

Estructura de la vegetación de las cercas vivas: Se registraron un total de 770 individuos pertenecientes a 34 especies arbóreas. En las cercas vivas simples se registraron un total de 13 especies y en las cercas vivas multiestrato
29 especies arbóreas. Las cercas multiestrato presentaron una mayor abundancia, riqueza, diversidad, dap, altura total, que las cercas vivas simples, pero no hubo diferencias en el diámetro de la copa (Cuadro 1).

La composición de especies en las cercas vivas multiestrato estuvieron dominadas por Bursera simaruba (Indio desnudo: 54\% de los individuos), Cordia alliodora (Laurel; $13 \%$ ), Tabebuia rosea (Roble; 5.2\%), Trichilia havanensis (Uruca; 4.8\%), Anacardium occidentale (Marañon; 3.7\%) y Entorolobium cyclocarpum (Guanacaste; 3\%), que equivalen el $83 \%$ del total de los individuos registrados en estas cercas. Las cercas vivas simples estuvieron dominadas por Bursera simaruba (Indio desnudo; $82 \%$ de los individuos) y Bombacopsis quinata (Pochote; 14\%).

Caracterización de las mariposas en las cercas vivas: Se registró un total de 2782 individuos pertenecientes a 75 especies de mariposas, las especies comunes en las cercas vivas fueron: Anartia fatima, Dryas julia, Eurema daria, E. nise, Hamadryas februa ferentina, Hermeuptychia hermes, Junonia evarete, Phoebis philea, Siproeta stelenes. Estas son

CUADRO 1

Comparación de medias del número de especies, abundancia, diversidad de Shannon, diámetro a la altura del pecho (dap), altura total y diámetro de copa, entre las cercas vivas simples (CVS) y cercas vivas multiestrato (CVM) en Esparza, Costa Rica, 2006

TABLE 1

Mean comparison for number of species, abundance, Shannon diversity, diameter at breast (dab), total height of trees and canopy radius among multi-strata (CVM) and simple (CVS) live fences in Esparza, Costa Rica, 2006

\begin{tabular}{|c|c|c|c|}
\hline Variable & CVM & CVS & $\mathrm{p}$ \\
\hline No. de especies & $9.2 \pm 2.28 \mathrm{a}$ & $4 \pm 1.22 \mathrm{~b}$ & 0.0010 \\
\hline Abundancia & $46.8 \pm 16.45 b$ & $107.2 \pm 54.61 \mathrm{a}$ & 0.0321 \\
\hline Shannon & $1.45 \pm 0.34 \mathrm{a}$ & $0.44 \pm 0.33 \mathrm{~b}$ & 0.0007 \\
\hline $\operatorname{dap}(\mathrm{cm})^{*}$ & $23.83 \pm 8.5 \mathrm{a}$ & $16.91 \pm 5.26 \mathrm{~b}$ & 0.0801 \\
\hline Altura total $(\mathrm{m})^{*}$ & $9.18 \pm 3.11 \mathrm{a}$ & $6.57 \pm 2.26 b$ & 0.0839 \\
\hline Diámetro de copa (m) & $8.27 \pm 3.43 \mathrm{a}$ & $5.97 \pm 2.32 \mathrm{a}$ & 0.1241 \\
\hline
\end{tabular}


especies con alta capacidad de dispersión y asociadas con hábitats perturbados (DeVries 1987). Las nueve especies comunes (Cuadro 2), representan el $75 \%$ del total de individuos observados durante el periodo de muestreo.

Comparación de la abundancia, diversidad y riqueza de especies de mariposas entre cercas vivas simples y multiestrato: En las cercas vivas multiestrato (CVM) se registró 1605 individuos pertenecientes a 70 especies de mariposas, comparado con 1177 individuos de 51 especies de mariposas en las cercas vivas simples (CVS; Anexo 1). En las CVM se registró el mayor número de individuos, especies, diversidad de Shannon que en las CVS (Cuadro 3), mientras que el índice de diversidad de Simpson mostró valores similares.

El índice de complementariedad entre las cercas vivas (CVM, CVS) fue del 38\%, y evidenció que la composición de especies de mariposas entre estos dos tipos de cercas es muy

CUADRO 2

Listado de las nueve especies más abundantes en las cercas vivas simples (CVS) y multiestrato (CVM), en Esparza Costa Rica, 2005-2006

TABLE 2

List of the nine most commonly observed species of butterflies in multi-strata live fences (CVM) and simple live fences (CVS) in Esparza, Costa Rica, 2005-2006

$\begin{array}{lccc}\text { Nombre científico } & \text { CVM (\%) } & \text { CVS (\%) } & \text { Total (\%) } \\ \text { Eurema daira daira Godart, 1819 } & 28.38 & 32.17 & 29.98 \\ \text { Hermeuptychia hermes Fabricius, 1775 } & 15.18 & 11.83 & 13.77 \\ \text { Anartia fatima Godart, 1824 } & 8.03 & 6.81 & 7.51 \\ \text { Phoebis philea philea Johansson, } & 5.10 & 6.13 & 5.54 \\ \text { Junonia evarete Cramer, 1775 } & 4.73 & 9.87 & 6.90 \\ \text { Eurema nise Cramer, 1775 } & 4.23 & 2.64 & 3.56 \\ \text { Hamadryas februa ferentina Godart, 1824 } & 3.36 & 1.79 & 2.70 \\ \text { Dryas julia } \text { Fabricius, 1775 } & 3.11 & 2.98 & 3.06 \\ \text { Siproeta stelenes Linnaeus, } 1758 & 2.74 & 2.47 & 2.62 \\ \text { Total } & 74.86 & 76.68 & 75.63\end{array}$

\section{CUADRO 3}

Comparación del número promedio de la abundancia, la riqueza y diversidad de mariposas registradas entre las cercas vivas simples (CVS) y cercas vivas multiestrato (CVM) en Esparza, Costa Rica, 2005-2006

TABLE 3

A comparison of mean abundance, richness and diversity of butterflies observed among the multi-strata (CVM) and simple (CVS) live fences in Esparza, Costa Rica, 2005-2006

$\begin{array}{lccc}\text { Hábitat } & \text { CVM }(\mathrm{n}=5) & \text { CVS }(\mathrm{n}=5) & \mathrm{p} \\ \text { Abundancia } & 321.4 \pm 4.18 \mathrm{a} & 235 \pm 8.92 \mathrm{~b} & <0.0001 \\ \text { No. de especies } & 36.8 \pm 0.97 \mathrm{a} & 27.6 \pm 1.89 \mathrm{~b} & 0.0025 \\ \text { Shannon } & 2.6 \pm 0.05 \mathrm{a} & 2.44 \pm 0.04 \mathrm{~b} & 0.0431 \\ \text { Simpson } & 0.14 \pm 0.02 \mathrm{a} & 0.15 \pm 0.01 \mathrm{a} & 0.7565\end{array}$

\footnotetext{
- Valores con el error estándar, Letras diferentes en la misma fila indican diferencias estadísticas significativas, prueba T $(\mathrm{p}<0.05)$.

- Values with standard deviation, different letters within the same row indicate statistically significant differences $(p<0.05)$ using a T-test.
} 
parecida. Adicionalmente, de las 75 especies, 46 especies fueron registradas en ambos sitios, cinco fueron exclusivas de CVS (Marpesia chiron, Adelpha basiloides, Callicore phiteas, Neographium epidaus, Nica flavilla), y 24 para las CVM, donde se observaron especies como Morpho peleides, Taygetis mermeria, T. kerea, T. xenana, Pierella luna, Siproeta epaphus, Melinaea ethra, que son especies dependientes de bosque (DeVries 1987), y pueden estar utilizando estos hábitats en el agropaisajes para realizar sus movimientos en las áreas de pasturas.

Las curvas de acumulación de especies alcanzaron una fase asintótica en ambos tipos de cercas (Fig. 1) y el estimador de riqueza indicó un nivel de inventario superior al 87\% para CVM y del $91 \%$ en las CVS (Cuadro 4). Asimismo, el estimador de riqueza predice que se pueden registrar hasta siete especies en el conjunto de ambos tipos de cercas en el paisaje estudiado.
Gremios alimenticios: Las 75 especies de mariposas se agruparon en dos gremios alimenticios, el gremio nectarívoro fue en ambos tipos de cercas el más abundante (Fig. 2), representando $58 \%$ en CVM y $63 \%$ en CVS, y el de generalistas, un $42 \%$ en CVM y $37 \%$ para CVS. La proporción de individuos fue dependiente del tipo de cerca viva $(\mathrm{G}=5.26$; $\mathrm{p}=0.0218$ ).

Relación entre la estructura y diversidad de la vegetación y la riqueza de mariposas: La relación entre las variables de la estructura de vegetación utilizadas (altura, dap, cobertura arbórea, número de árboles, riqueza de especies y número de arbustos) y la riqueza de especies de mariposas, evidenció en el agropaisaje de Esparza que el número de especies arbóreas $(\mathrm{p}=0.03 ; \mathrm{AIC}=7.980)$ y el número de arbustos $(\mathrm{p}<0.001 ; \mathrm{AIC}=2.647)$, fueron las variables que más incidieron en la diversidad de mariposas que usan estos hábitats para

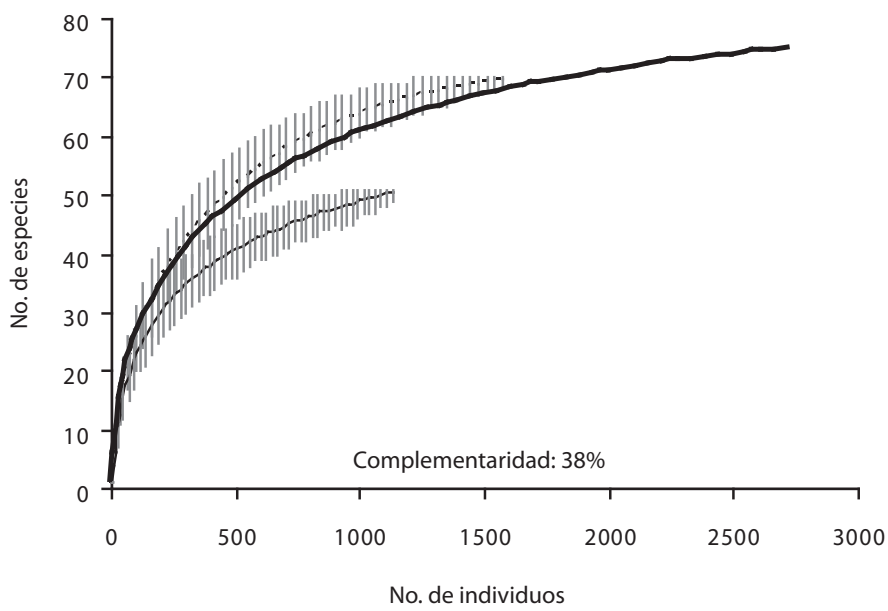

Fig. 1. Curva de acumulación de especies de mariposas entre las cercas vivas multiestrato (línea discontinua; $n=1605$ ), cercas vivas simples (línea continua; $\mathrm{n}=1177$ ), y ambos hábitat (línea gruesa; $\mathrm{n}=2782$ ) Esparza, Costa Rica, 2005-2006. Las líneas verticales representan los intervalos de confianza al 95\%. El valor de complementariedad expresa la diferencia de composición de la lista de especies de los dos tipos de cercas vivas (Colwell \& Coddington 1994).

Fig. 1. Butterfly species accumulation curve among multi-strata live fences (dotted line; $\mathrm{n}=1$ 605), simple live fences (continuous line; $n=1$ 177) in Esparza, Costa Rica, 2005-2006. The vertical lines represent 95\% confidence intervals. The complementary value is the difference in composition among the list of species in both types of live fences (Colwell \& Coddington 1994). 


\section{CUADRO 4}

Riqueza de especies observada y estimada de mariposas, usando el estimador de riqueza de segundo orden de CHAO y el modelo de Clench, en las cercas vivas multiestrato (CVM) y simples (CVS) en Esparza, Costa Rica

TABLE 4

Observed and estimated butterfly species richness, using the Clench model and the CHAO richness estimator, on multi-strata (CVM) and simple (CVS) live fences in Esparza, Costa Rica

$\begin{array}{lccc} & \text { CVM } & \text { CVS } & \text { Total } \\ \text { Especies observadas } & 70 & 51 & 75 \\ \text { Modelo de Clench } & 80 & 56 & 82 \\ \text { Chao 2 } & 84 & 64 & 94 \\ \text { Singletones } & 9 & 12 & 10 \\ \text { Dobletones } & 13 & 5 & 10 \\ \text { Nivel de Inventario } & 83.3-87.5 & 79.6-91.0 & 79.8-91.5 \\ \text { IC 95\% } & 62.9-77.1 & 44.2-57.8 & 66.9-83.1\end{array}$

- El nivel de inventario es el porcentaje de la riqueza de especies estimada (mínimo-máximo). IC: medias simuladas con los intervalos de confianza al 95\% del número de especies esperadas, basadas en 1000 simulaciones. Medias son consideradas diferentes si los intervalos de confianza no se traslapan.

- The inventory level is the estimated species richness percentage (minimum-maximum), and the IC is the simulated mean with $95 \%$ confidence intervals of expected number of species based on 1000 simulations. Means are considered different if confidence intervals do not overlap.

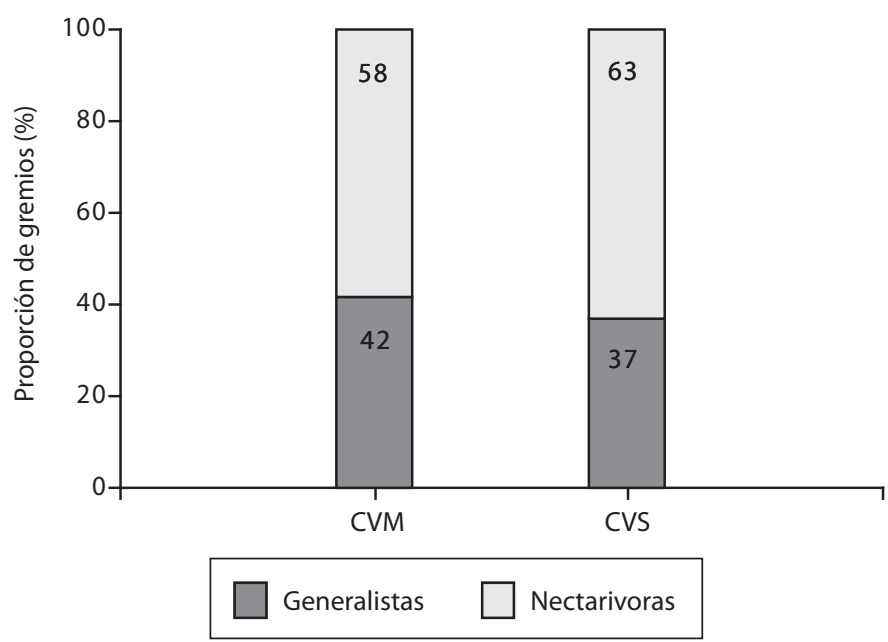

Fig. 2. Proporción de gremios alimenticios de mariposas (generalistas y nectarívoras) en las cercas vivas multiestrato (CVM) y simples (CVS). Los números en las barras indican la proporción de individuos que pertenecieron a cada gremio.

Fig 2. Proportion (\%) of butterfly guilds (generalists and nectarivorous) among multi-strata live fences (CVM) and simple live fences (CVS). The numbers on the bars indicate the ratio of individuals that belong to each guild. 
realizar sus movimientos en el paisaje, estas variables explican la variación de la diversidad de mariposas en un 55\% (Fig. 3). La riqueza de mariposas y el número de arbustos, presentó un ajuste cuadrático, generó un óptimo de 7 arbustos, para 41 especies de mariposas por cada $200 \mathrm{~m}$ de longitud de cercas vivas con diferentes estratos (Fig. 4a). La riqueza de mariposas y el número de especies arbóreas fue lineal y significativa (Fig. 4b).

\section{DISCUSIÓN}

Las cercas vivas multiestrato (CVM) presentaron una mayor complejidad estructural y diversidad de especies arbóreas que las cercas vivas simples (CVS), este resultado ha sido similar al encontrado en estudios realizados en otros paisajes agropecuarios evaluados en Centro América (Cárdenas et al. 2003; Lang et al. 2003; Ramírez 2007). Estas pueden constituir un hábitat que contribuye a minimizar el impacto de la generación de pasturas o área productivas y pueden jugar un papel para la conservación de las mariposas en paisajes agropecuarios.

Las CVM es un hábitat que presentó una mayor riqueza, abundancia de mariposas que las CVS, esto puede ser debido a que algunas especies de mariposas pueden estar utilizando las cercas vivas para realizar sus movimientos (alimentación, cópula, postura de huevos, protección contra depredadores, entre otros) a través del paisaje agropecuario. La importancia de CVM y cortinas rompevientos para la conservación de la fauna silvestre ha sido apreciada en otros paisajes principalmente para aves (Cárdenas et al. 2003, Lang, et al. 2003, Vílchez et al. 2004, 2008), mamíferos (Harvey et al. 2006, Medina et al. 2006), pocos estudios se han realizado con insectos (Hernández et al. 2003, Tobar et al. 2007, Mendoza et al. 2008).

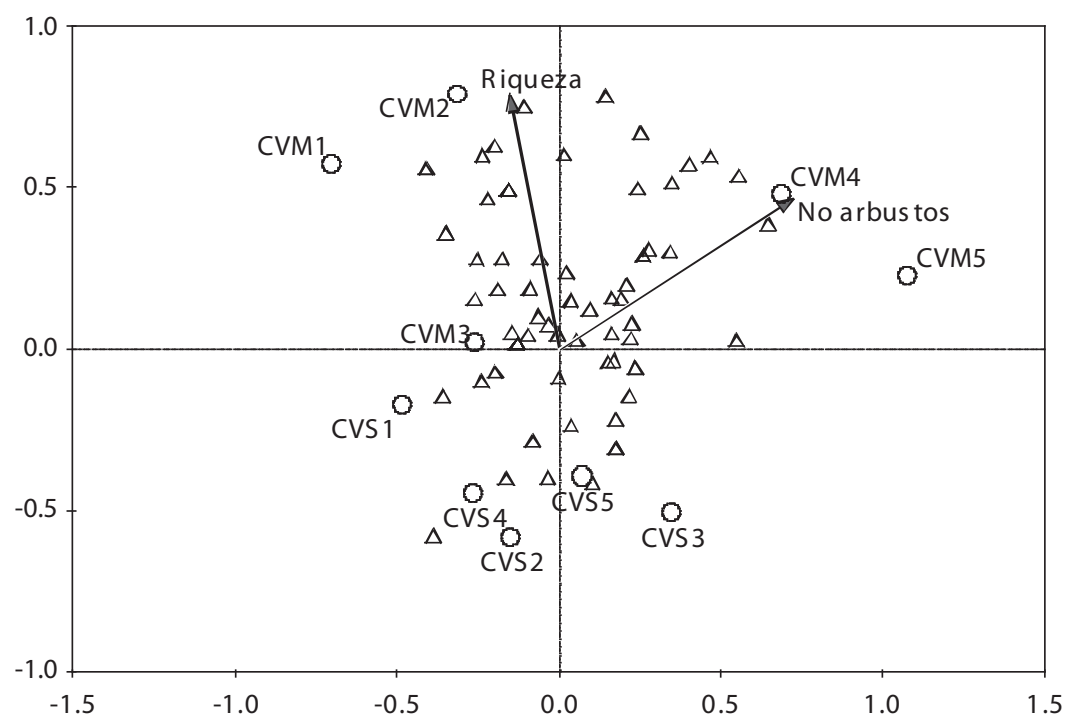

Fig. 3. Análisis de correspondencia rectificado (DCA) para la fauna de mariposas de Esparza, Costa Rica. 2005-2006. Los círculos son los transectos establecidos en las cercas vivas multiestrato (CVM) y simples (CVS). Los triángulos representan las especies de mariposas. Las flechas son las variables que explican la riqueza de mariposas en estos hábitats.

Fig. 3. Detrended Correspondence Analysis (DCA) for butterfly communities in Esparza, Costa Rica, 2005-2006. The circles represent the transects established in each type of live fence. The triangles represent the species of butterflies. The arrows are the variables that explain the richness of butterflies in these habitats. CVM: multi-strata live fence; CVS: simple live fence. 

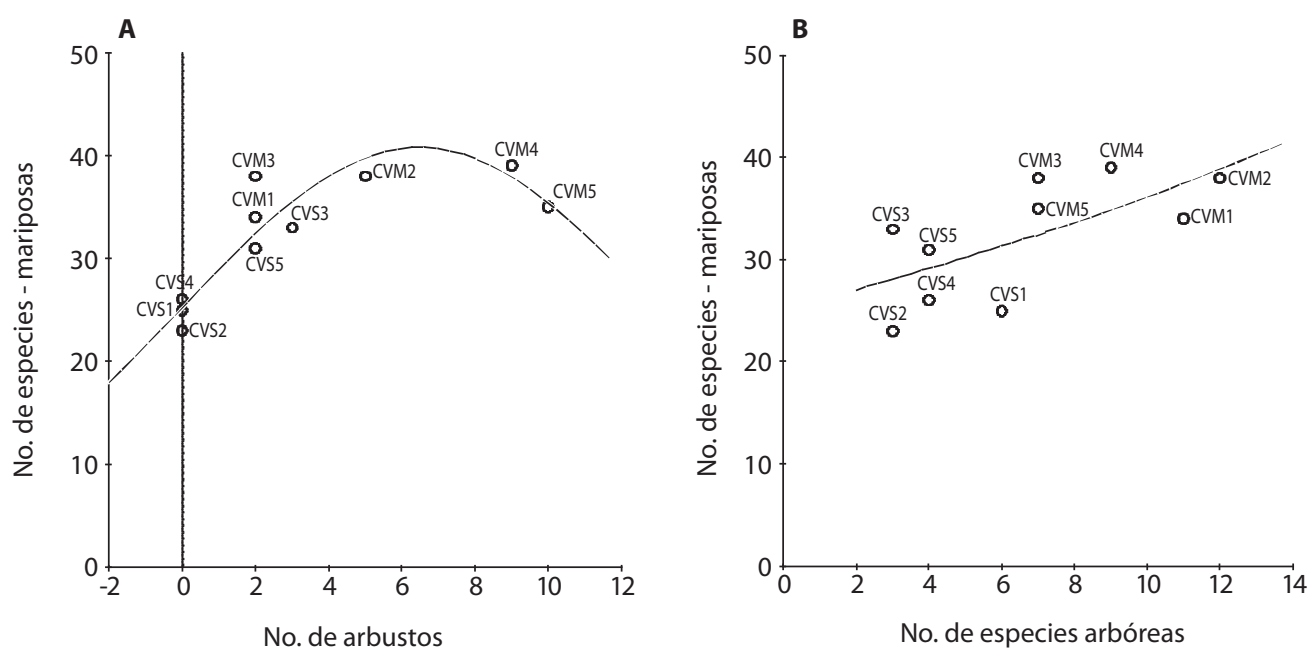

Fig. 4. Relación entre la riqueza de mariposas y (A) el número de arbustos y (B) el número de especies arbóreas en cercas vivas presentes en Esparza, Costa Rica. 2005-2006. CVM: cerca viva multiestrato y CVS: cerca vivas simples.

Fig. 4. Relationship between butterfly species richness according to (A) number of shrubs and (b) number of tree species presents in live fences in Esparza, Costa Rica, 2005-2006. CVM: multi-strata live fences; CVS: simple live fences.

El análisis del índice de diversidad de Shannon fue mayor en las CVM que en CVS, mientras que el índice de Simpson no presentó diferencias, lo cual puede estar relacionado con las nueve especies más comunes que representaron el 74.86 y $76.68 \%$ del total de individuos en cercas complejas y simples, respectivamente (Cuadro 2).

De las 75 especies presentes en ambas cercas, el $93 \%$ fueron observadas en CVM y el $68 \%$ en CVS, lo que puede indicar que ambos tipos de cercas pueden proveer fuentes alimenticias y nichos para el mantenimiento de la diversidad de mariposas en el paisaje agropecuario. El gremio nectarívoro fue dominante en ambos tipos de cercas, debido a que estos dependen de los recursos florísticos que están asociados a las cercas y pasturas de la región, los cuales son necesarios para la supervivencia de este grupo en el agropaisaje (Tobar et al. 2001, Ouin et al. 2004).

Las diferencias entre la riqueza y composición de especies de mariposas $(\mathrm{C}=32 \%)$, se puede deber a que en las CVM se registró la mayoría de mariposas dependientes de bosque, que por lo general son especies que vuelan en el sotobosque y dosel de los remanentes de bosque que persisten dentro del paisaje agropecuario. Sin embargo, algunas de ellas fueron escasas o estuvieron ausentes en las CVS, lo que puede estar relacionado con diversidad de árboles y arbustos presentes en las cercas vivas, y con las actividades de manejo que realizan los productores en las CVS para el mantenimiento de la pastura, mediante el uso de herbicidas, la frecuencia de podas y chapeas, que ocasionan cambios en la composición, alteración en el crecimiento y diversidad vegetal (Kruess \& Tscharntke 2002), que impiden la creación de hábitats adecuados para el mantenimiento $\mathrm{y}$ conservación de especies de mariposas en el paisaje agropecuario. Por otra parte, las CVM aunque tienen árboles que podan, retienen especies arbóreas y arbustivas provenientes de la regeneración natural y son utilizadas para producción de frutas, leña, sombra, forraje en las fincas (Villanueva et al. 2007), y pueden ayudar a mantener el $37 \%$ de las especies de 
mariposas dependientes de las áreas boscosas en el agropaisaje (Tobar et al. 2006). Por lo tanto, el diseño y manejo correcto de estos hábitats, constituyen elementos importantes para la conservación de mariposas en el paisaje agropecuario.

Esas diferencias también pueden estar relacionas con la riqueza de especies de árboles y arbustos asociadas a las CVM, y que pueden ofrecer una variedad de recursos alimenticios (flores, frutos, excrementos), plantas hospederas, sitios de descanso o percha, además de reducir el efecto negativo de la formación de pastizales en monocultivos sobre las poblaciones de mariposas (Daily \& Erlich 1996, Harvey et al. 2005). Adicionalmente, pueden servir como corredores para permitir el movimiento de varias especies de mariposas a través del paisaje (Haber \& Stevenson 2004; Harvey et al. 2008) y pueden facilitar la migración altitudinal que realizan algunas especies de mariposas durante la época seca (noviembre-mayo) hacia ambientes más húmedos $\mathrm{y}$ frescos en los alrededores de las montañas de Tilarán (Monteverde-Costa Rica) o hacia la región Atlántica. Haber \& Stevenson (2004) estimaron que en la región Pacifico Norte y Central del Pacifico, migran entre el 40 y $75 \%$ de las especies, y apreciaron la migración de algunas especies de mariposas a Monteverde como: Ascia monuste, Aphrissa statira, Eurema spp., Phoebis spp., Smyrna blomfilda, Siproeta stelenes, Euptoieta hegesia, Adelpha spp., las cuales fueron registradas en las cercas vivas en la región de Esparza.

En compendio, las cercas vivas son importantes para la conservación de la diversidad de lepidópteros en los paisajes agropecuarios, la cual está relacionada con el número de arbustos y riqueza de especies arbóreas que puedan presentar. Estos elementos estructurales, en conjunto con las áreas de remanentes de bosque, pueden jugar un papel importante en la conservación de la diversidad de los paisajes agropecuarios, además, de servir como corredores para los movimientos migratorios altitudinales que realizan durante la época seca.

\section{AGRADECIMIENTOS}

Los autores agradecen al equipo del proyecto GEF-SSP por su apoyo para la realización de esta investigación, a los productores de la región de Esparza por permitir el ingreso a sus fincas. A Fernando Casanoves, y Jeffrey Milder por los comentarios al manuscrito final. Esta investigación se realizó como parte del proyecto "Enfoques silvopastoriles integrados para el manejo de ecosistemas, GEF-SSP, liderado por el CATIE, Nitlapan y CIPAV. Los autores son responsables del material divulgado en este artículo; esta publicación no representa la opinión de GEF.

\section{RESUMEN}

En Centro América, los paisajes agropecuarios mantienen diferentes formas de cobertura arbórea como árboles dispersos, cercas vivas, fragmentos de bosque, bosques ribereños, que pueden generar hábitats apropiados para la conservación de la biodiversidad. El presente estudio caracterizó la composición, riqueza y abundancia de mariposas en dos tipos de cercas vivas presentes en un paisaje dominado por pasturas en el Pacifico Central de Costa Rica. Se seleccionaron un total de cinco cercas vivas por cada tipo de cerca (simples y multiestrato), donde se establecieron franjas de $120 \times 5 \mathrm{~m}$ que fueron recorridos por espacio de una hora durante dos días/hábitat. Se registró un total de 2782 individuos, pertenecientes a 75 especies de mariposas. Las especies más abundantes fueron: Anartia fatima, Eurema daría, E. nise, Hermeuptychia hermes, Junonia evarete y Phoebis philea. Las cercas vivas multiestrato presentaron una mayor riqueza y abundancia de especies de mariposas que las cercas vivas simples. Las cercas vivas multiestrato pueden ayudar a mantener el $56 \%$ de las especies encontradas en los bosques secundarios y ribereños. Este tipo de cerca viva pueden jugar un papel importante para la conservación de mariposas en áreas de producción pecuaria, y su beneficio está influenciado por el tipo manejo que realizan los productores.

Palabras claves: Biodiversidad, cercas vivas, Costa Rica, fragmentación, hábitat, gremios, Papilionoidea, sistemas silvopastoriles, servicios ambientales, riqueza de especies.

\section{REFERENCIAS}

Balmford, A. \& W. Bond. 2005. Trends in the state of nature and their implications for human well-being. Ecol. Lett. 8:1218-1234 
Beer, J., C.A. Harvey, M. Ibrahim, J.M. Harmand, E. Somarriba \& F. Jimenez. 2003. Servicios ambientales de los sistemas agroforestales. Agroforestería Américas 10:80-87.

Bennett, A. 1999. Linkages in the landscape: the role of corridors and connectivity in wildlife conservation. UICN. Gland, Suiza.

Cárdenas, G., C.A. Harvey, M. Ibrahim \& B. Finegan. 2003. Diversidad y riqueza de aves en diferentes hábitats en un paisaje fragmentado en Cañas, Costa Rica. Agroforestería Américas 10: 78-85.

Casasola, F., M. Ibrahim, C. Sepúlveda, N. Ríos \& D. Tobar. 2009. Implementación de sistemas silvopastoriles y el pago de servicios ambientales en Esparza, Costa Rica: una herramienta para la adaptación al cambio climático en fincas ganaderas, p. 169-188. In M. Ibrahim \& C. Sepúlveda (eds). Políticas y sistemas de incentivos para el fomento y adopción de buenas prácticas agrícolas como una medida de adaptación al cambio climático en América Central. Centro Agronómico Tropical (CATIE). Turrialba, Costa Rica.

Chacón, I. \& J. Montero. 2007. Mariposas de Costa Rica. Instituto Nacional de Biodiversidad INBIO, Santo Domingo de Heredia, Costa Rica.

Chacón-L, M. \& C.A. Harvey. 2008. Contribuciones de las cercas vivas a la estructura y la conectividad de un paisaje fragmentado en Río Frío, Costa Rica, p. 225-250. In C.A. Harvey \& J.C. Sáenz (Eds). Evaluación y conservación de biodiversidad en paisajes fragmentados de Mesoamérica. Instituto Nacional de Biodiversidad INBIO. Santo Domingo de Heredia, Costa Rica.

Colwell, R.K. \& J.A. Coddington. 1994. Estimating terrestrial biodiversity through extrapolation. Philos. Trans. R. Soc. Lond. B. Biol. Sci. 345: 101-118.

D’Abrera, B. 1981. Butterflies of the Neotropical Region. Part I Papilionidae and Pieridae. Hill House. Victoria, Australia.

D’Abrera, B. 1984. Butterflies of the Neotropical Region. Part II Danaidae, Ithomiidae, Heliconidae \& Morphidae. Hill House. Londres, Reino Unido.

D’Abrera, B. 1987a. Butterflies of the Neotropical Region. Part III Brassolidae, Acraeidae, Nymphalidae (part I) Hill House.Victoria, Australia.

D’Abrera, B. 1987b. Butterflies of the Neotropical Region. Part IV Nymphalidae (Part II). Hill House. Victoria, Australia.
D'Abrera, B. 1989. Butterflies of the Neotropical Region. Part V Nymphalidae (Conc.) and Satyridae. Hill House. Victoria, Australia.

D’Abrera, B. 1994. Butterflies of the Neotropical Region. Part VI Riodinidae. Hill House. Victoria, Australia.

D’Abrera, B. 1995. Butterflies of the Neotropical Region. Part VII Lycaenidae. Hill House. Victoria, Australia.

Daily, G.C. \& P.R. Ehrlich. 1996. Nocturnality and species survival. Proc. Natl. Acad. Sci. 93: 11709-11712.

Daily, G.C., P.R. Ehrlich \& G.A. Sánchez-Azpfeita. 2001. Countryside biogeography: utilization of human dominated habitats by the avifauna of southern Costa Rica. Ecol. Appl. 11: 98-108.

Devendra, C. \& M. Ibrahim. 2004. Silvopastoral systems as a strategic for diversification and productivity enhancement from Livestock in the tropics, p. 10-24 In L. Mannetje, M. Ramirez, M. Ibrahim, C. Sandoval, N. Ojeda \& J. Ku (eds). The Importance of silvopastoril systems in rural livelihoods to provide ecosystem services. Second International Symposium of Silvopastoril Systems. Mérida, Yucatan, México.

DeVries, P.J. 1987. The butterflies of Costa Rica and their natural history: volumen I Papilionidae, Pieridae, Nymphalidae. Princeton University. Nueva Jersey, EEUU.

DeVries, P.J. 1997. Butterflies of Costa Rica and their natural history: volume II (Riodinidae). Princeton University. Nueva Jersey, EEUU.

Estrada, A. \& R. Coates-Estrada. 2002. Dung beetles in continuous forest, forest fragments and in an agricultural mosaic habitat island at Los Tuxtlas, Mexico. Biodivers. Conserv. 11: 1903-1918.

Estrada, A. \& R. Coates-Estrada. 2005. Diversity of Neotropical migratory land bird species assemblages in forest fragments and man-made vegetation in Los Tuxtlas, Mexico. Biodivers. Conserv. 14: 1719-1734.

FAO (Organización de las Naciones Unidas para la Agricultura y la Alimentación). 2003. Situación de los bosques del mundo. Roma, Italia.

Guevara, S., J. Laborde \& G. Sánchez. 1998. Are isolated remnant trees in pastures a fragmented canopy? Selbyana $19: 34-43$.

Guevara, S., J. Laborde \& G. Sánchez-Ríos. 2005. Los árboles que la selva dejó atrás. Interciencia 30: 595601 . 
Harber, W.A. \& R.D. Stevenson. 2004. Diversity, migration, and conservation in Northern Costa Rica, p. 99-114. In G.W. Frankie, A. Mata \& S. Vinson (eds). Biodiversity conservation in Costa Rica: learning the lessons in a seasonal dry forest. University of California. Londres, Reino Unido.

Harvey, C.A. \& W.A. Haber. 1999. Remnant trees and the conservation of biodiversity in Costa Rican pastures. Agroforest. Syst. 44: 37-68.

Harvey, C.A., C.F. Guindon, W.A. Harber, D. Hamilton \& K.G. Murray. 2008. Importancia de los fragmentos de bosque, los árboles dispersos y las cortinas rompevientos para la biodiversidad local y regional de Monteverde, Costa Rica, p. 289-326. In C.A. Harvey \& J.C. Sáenz (eds). Evaluación y conservación de biodiversidad en paisajes fragmentados de Mesoamérica. Instituto Nacional de Biodiversidad INBIO, Santo Domingo de Heredia, Costa Rica.

Harvey, C., A. Medina, D. Sanchez, S. Vilchez, B. Hernandez, J. Saenz, J.M. Maes, F. Casanoves \& F.L. Sinclair. 2006. Patterns of Animal Diversity in Different Forms of Tree Cover in Agricultural Landscapes. Ecol. Appl. 16: 19-86.

Harvey, C.A., C. Villanueva, J. Villacis, M. Chacón, D. Muñoz, M. Ibrahim, R. Gómez, M. López, R. Taylor, J. Martínez, A. Navas, J. Saenz, D. Sánchez, A. Medina, S. Vilchez, B. Hernández, A. Pérez, E. Ruiz, L. Lang \& F.L. Sinclair. 2005. Contribution of live fences to the ecological integrity of agricultural landscapes. Agr. Ecosys. Environ. 111: 200-230.

Hernández, B., J.M. Maes, C.A. Harvey, S. Vílchez, A. Medina \& D. Sánchez. 2003. Abundancia y diversidad de escarabajos coprófagos y mariposas diurnas en un paisaje ganadero en el departamento de Rivas, Nicaragua. Agroforestería Américas 10: 93-102.

Holdridge, L.R. 1963. Life zone ecology. Costa Rica, IICA. San José, Costa Rica.

Ibrahim, M., S. Abarca \& O. Flores. 2000. Geographical synthesis of data on Costa Rica pastures and their potential for improvement, p. 423-448. In Hall C. (ed) Quantifying sustainable development. The future of tropical economies. Academic. San Diego, EEUU.

InfoStat. 2007. InfoStat, versión 2007. Manual del Usuario. Grupo InfoStat, FCA, Universidad Nacional de Córdoba. Primera Edición, Editorial Brujas, Córdoba, Argentina.

Kaimowitz, D. 2001. Will livestock intensification help save Latin America tropical forest? p. 1-20. In A. Angelsen \& D. Kaimowitz (eds). Agricultural technologies and tropical deforestation. CABI. Wallingford, Reino Unido.

Kallenbach R.L., M.S. Kerley \& G.J. Bishop-Hurley. 2006. Cumulative forage production, forage quality and livestock performance from an annual ryegrass and cereal rye mixture in a Pine-Walnut Silvopasture. Agroforest. Syst. 66: 43-53.

Kruess, A. \& T. Tscharntke. 2002. Grazing intensity and the diversity of grasshoppers, butterflies, and trap-nesting bees and wasps. Conserv. Biol. 16:1570-1580.

Lamas, G (ed). 2004. Checklist: Part 4a. Hesperioidea; Papilionoidea. Atlas of Neotropical Lepidoptera Vol.5a. Association for Tropical Lepidoptera. Scientific Publishers. Gainesville, Florida, EEUU.

Lang, I., L.H. Gormley, C.A. Harvey \& F.L. Sinclair. 2003. Composición de la comunidad de aves en cercas vivas del Río Frío, Costa Rica. Agroforestería Américas 10: 86-92.

Magurran, A. 2003. Measuring biological diversity. Blackwell. Oxford. Reino Unido.

Mcgeoch, M.A., B.J. Van Rensburg \& A. Botes. 2002. The verification and application of bioindicators: a case study of dung beetles in a savanna ecosystem. Ecol. Appl. 39: 661-672.

Medina, A., C.A. Harvey, D. Sanchez, S. Vilchez \& B. Hernandez. 2006. Bat diversity and movement in an agricultural landscape in Matiguás, Nicaragua. Biotropica 39: 120-128.

Mendoza, J.E., E. Jiménez, F.H. Lozano-Zambrano, P. Caycedo-Rosales \& L.M. Rengifo. 2008. Identificación de elementos del paisaje prioritarios para la conservación de biodiversidad en paisajes rurales de los Andes Centrales de Colombia, p. 251-288. In C.A. Harvey \& J.C. Sáenz (Eds). Evaluación y conservación de biodiversidad en paisajes fragmentados de Mesoamérica. Instituto Nacional de Biodiversidad INBIO. Santo Domingo de Heredia, Costa Rica.

Murcia, C. 1995. Edge effects in fragments: implications for conservation. Tree 10: 58-62.

Ouin, A., S. Aviron, J. Vover \& F. Burel. 2004. Complementation/suplementation of resources for butterflies in agricultural landscapes. Agr. Ecosyst. Environ. 103: 473-479.

Pagiola S., P. Angostini, J. Gobbi, M. Ibrahim, E. Murgueitio, E. Ramírez, M. Rosales \& J.P. Ruiz. 2004. Paying for biodiversity conservation services in agricultural landscapes. Paper 96. Departamento Ambiental, Banco Mundial. Washington DC, EEUU. 
Petit, L.J. \& D.R. Petit. 2003. Evaluating the importance of human-modified lands for neotropical bird conservation. Conserv. Biol. 17: 687-694.

Pezo, D. \& M. Ibrahim. 1998. Sistemas silvopastoriles. Módulo de Enseñanza Agroforestal No. 2. CATIEGTZ. Turrialba, Costa Rica.

Ramírez, L. 2007. Contribución ecológica y cultural de los sistemas silvopastoriles para la conservación de la biodiversidad en Matiguás, Nicaragua [Tesis de Maestría]. CATIE. Turrialba, Costa Rica.

Ríos, N., A. Cárdenas, H. Andrade, M. Ibrahim, F. Jimenez, F. Sancho, E. Ramírez, B. Reyes \& A. Woo. 2007. Escorrentía superficial e infiltración en sistemas ganaderos convencionales y silvopastoriles en el trópico subhúmedo de Nicaragua y Costa Rica. Agroforestería Américas 45: 66-71.

Sáenz, J.C., F. Villatoro, M. Ibrahim, D. Fajardo \& M. Pérez. 2007. Relación entre las comunidades de aves y la vegetación en agropaisajes dominados por la ganadería en Costa Rica, Nicaragua y Colombia. Agroforestería Américas 45: 37-48.

Soberón, J.M. \& J.B. Llorente. 1993. The use of species accumulation functions for the prediction of species richness. Conserv. Biol. 7: 480-488.

Somarriba, E. 1992. Revisiting the past: an essay on agroforestry definition. Agroforest. Syst. 19: 233-240.

Ter Braak, C.J.F. \& P. Smilauer. 2002. CANOCO Reference manual and software for canonical community ordination (Version 4.5). Wageningen University and Research Centre. Wageningen, Paises Bajos.

Tobar-L, D.E., J.O. Rangel-CH \& G. Andrade-C. 2001. Las cargas polínicas de las mariposas (Lepidoptera: Rophalocera) de la parte alta de la cuenca del río Roble, Quindío, Colombia. Caldasia 23: 549-557.
Tobar, D., M. Ibrahim, C. Villanueva \& F. Casasola. 2006. Diversidad de mariposas diurnas en un paisaje agropecuario en la región del Pacifico Central, Costa Rica. p. 25-35. In Memorias-IV Congreso Latinoaméricano de Agroforestería, 24-28 de octubre- Centro de Convenciones „Plaza América“. Varadero, Cuba.

Tobar, D., M. Imbrahim \& F. Casasola. 2007. Diversidad de mariposas diurnas en un paisaje agropecuario en la región Pacifica Central de Costa Rica. Agroforestería Américas 45: 58-65.

Vílchez, S., C.A. Harvey, D. Sánchez, A. Medina \& B. Hernández. 2004. Diversidad de aves en un paisaje fragmentado de bosque seco en Rivas, Nicaragua. Encuentro 68: 24-48.

Vílchez, S., C.A. Harvey, D. Sánchez, A. Medina, B. Hernández \& R. Taylor. 2008. Diversidad y composición de aves en un agropaisaje de Nicaragua, p. 547-578. In C.A. Harvey \& J.C. Sáenz (eds). Evaluación y conservación de biodiversidad en paisajes fragmentados de Mesoamérica. Instituto Nacional de Biodiversidad INBIO, Santo Domingo de Heredia, Costa Rica.

Villanueva, C., D. Tobar, M. Ibrahim, F. Casasola, J. Barrantes \& R. Arguedas. 2007. Arboles dispersos en pasturas de fincas ganaderas en la región del Pacifico Central de Costa Rica. Agroforestería Américas 47:12-20

Zhang, H., A. Henderson-Sellers \& K. Mcguffie. 2001. The compounding effects of tropical deforestation and greenhouse warming on climate. Climatic Change 49: 309-338.

\section{REFERENCIAS DE INTERNET}

Colwell, R.K. 2004. ESTIMATES: Statistical estimation of species richness and shared species from samples. Version 7.0. (Consultado: 15 de marzo de 2007, http://viceroy.eeb.econn.edu/eESTIMATES). 


\section{ANEXO 1}

Listado taxonómico de las mariposas y abundancia relativa (\%) registradas en las cercas vivas multiestrato (CVM) y simples (CVS), en Esparza, Costa Rica. 2005- 2006.

ANNEX 1

Butterfly taxonomic list and relative abundance (\%) in multi-strata live fences (CVM) and simple live fences (CVS) observed in Esparza, Costa Rica. 2005-2006

\begin{tabular}{|c|c|c|c|}
\hline \multirow{2}{*}{ Especies } & \multicolumn{2}{|c|}{ Habitat } & \multirow{2}{*}{ Total } \\
\hline & CVM & CVS & \\
\hline \multicolumn{4}{|l|}{ Familia Papilionidae } \\
\hline \multicolumn{4}{|l|}{ Subfamilia Papilioninae } \\
\hline Battus polydamas polydamas Linnaeus, 1758 & 0.87 & 1.53 & 1.15 \\
\hline Heraclides thoas nealces Rothschild \& Jordan, 1905 & 0.25 & 0.34 & 0.29 \\
\hline Neographium epidaus epidaus Doubleday, 1846 & 0.00 & 0.09 & 0.04 \\
\hline Parides iphidamas iphidamas Fabricius, 1793 & 0.19 & 0.26 & 0.22 \\
\hline Parides montezuma Westwood, 1842 & 0.12 & 0.00 & 0.07 \\
\hline \multicolumn{4}{|l|}{ Familia Pieridae } \\
\hline \multicolumn{4}{|l|}{ Subfamilia Coliadinae } \\
\hline Anteos clorinde Godart, 1823 & 0.12 & 0.00 & 0.07 \\
\hline Aphrissa statira statira Cramer, & 0.25 & 0.26 & 0.25 \\
\hline Eurema albula Cramer, 1775 & 0.31 & 0.60 & 0.43 \\
\hline Eurema arbela boisduvaliana C \& R Felder, 1865 & 0.12 & 0.00 & 0.07 \\
\hline Eurema daira eugenia Wallengren, 1860 & 28.38 & 32.17 & 29.98 \\
\hline Eurema dina westwoodi Boisduval, 1836 & 0.81 & 0.00 & 0.47 \\
\hline Eurema lisa Boisduval \& Leconte, 1829 & 0.12 & 0.00 & 0.07 \\
\hline Eurema nise nelphe R. Felder, 1869 & 4.23 & 2.64 & 3.56 \\
\hline Eurema proterpia Fabricius, 1775 & 0.12 & 0.34 & 0.22 \\
\hline Eurema salome C \& R Felder, 1861 & 0.12 & 0.00 & 0.07 \\
\hline Phoebis philea philea Linnaeus, 1763 & 5.10 & 6.13 & 5.54 \\
\hline Phoebis sennae sennae Linnaeus, 1758 & 1.31 & 0.77 & 1.08 \\
\hline Phoebis trite trite Linnaeus, 1758 & 0.19 & 0.09 & 0.14 \\
\hline \multicolumn{4}{|l|}{ Subfamilia Pierinae } \\
\hline Ascia monuste Linnaeus, 1764 & 0.37 & 0.17 & 0.29 \\
\hline Ganyra josephina josepha Godman \& Salvin, 1868 & 0.12 & 0.00 & 0.07 \\
\hline Itaballia demophile Linnaeus, 1758 & 0.25 & 0.00 & 0.14 \\
\hline Leptophobia aripa aripa Boisduval, 1836 & 0.68 & 0.77 & 0.72 \\
\hline \multicolumn{4}{|l|}{ Familia Lycaenidae } \\
\hline \multicolumn{4}{|l|}{ Subfamilia Polyommatinae } \\
\hline Hemiargus hanno Stoll, 1790 & 1.49 & 5.02 & 2.98 \\
\hline \multicolumn{4}{|l|}{ Subfamilia Theclinae } \\
\hline Electrostrymon sp. Clench, 1961 & 2.12 & 0.77 & 1.55 \\
\hline Pseudolycaena marsyas Linnaeus, 1758 & 0.06 & 0.00 & 0.04 \\
\hline \multicolumn{4}{|l|}{ Familia Riodinidae } \\
\hline \multicolumn{4}{|l|}{ Subfamilia Riodininae } \\
\hline Calephelis browni McAlpine, 1971 & 0.19 & 0.00 & 0.11 \\
\hline Calephelis fulmen Stichel, 1910 & 0.19 & 0.00 & 0.11 \\
\hline Calephelis schausi McAlpine, 1971 & 0.12 & 0.26 & 0.18 \\
\hline
\end{tabular}




\section{ANEXO 1 (Continuación)}

Listado taxonómico de las mariposas y abundancia relativa (\%) registradas en las cercas vivas multiestrato (CVM) y simples (CVS), en Esparza, Costa Rica. 2005- 2006.

ANNEX 1 (Continued)

Butterfly taxonomic list and relative abundance (\%) in multi-strata live fences (CVM) and simple live fences (CVS) observed in Esparza, Costa Rica. 2005-2006

\section{Especies \\ Familia Nymphalidae \\ Subfamilia Danainae}

Danaus eresimus Cramer, 1777

Danaus plexippus Linnaeus, 1758

Mechanitis polymnia isthmia $\mathrm{H}$. W. Bates, 1863

Melinaea ethra maeonis Hewitson,

Tithorea harmonia Cramer, 1777

\section{Subfamilia Morphinae}

Morpho peleides peleides Kollar, 1850

Caligo memnon memnon C \& R Felder, 1866

\section{Subfamilia Satyrinae}

Cissia confusa Staudinger, 1888

Cissia similis Butler, 1867

Euptychia weswoodii Butler, 1863

Hermeuptychia hermes Fabricius, 1775

Magneuptychia libye Linnaeus, 1767

Pareuptychia hesione Sulzer, 1776

Pierella luna luna Fabricius,

Taygetis kerea Butler, 1879

Taygetis mermeria Cramer, 1779

Taygetis xenana Butler, 1870

Subfamilia Heliconiinae

Dryas julia Fabricius, 1775

Euptoieta hegesia Cramer, 1779

Agraulis vanillae Linnaeus, 1758

Heliconius erato petiveranus Doubleday, 1847

Heliconius melponeme rosina Boisduval,

Heliconius charitonius Linnaeus, 1767

Heliconius hecalesia formosus H. W. Bates, 1863

Dione moneta Hübner, 1819

Eueides isabella Cramer, 1780

\section{Subfamilia Limenitidinae}

Adelpha fessonia Hewitson, 1847

Adelpha basiloides H. W. Bates, 1865

Subfamilia Cyrestinae

Marpesia chiron Fabricius, 1775

\section{CVM}

Habitat

0.31

0.00

0.18

1.06

1.19

1.11

0.12

0.09

0.11

0.19

0.00

0.11

0.06

0.00

0.04

0.25

0.00

0.14

0.06

0.09

0.07

0.75

0.09

0.47

0.68

1.36

0.97

1.43

15.18

0.68

1.11

2.43

11.83

13.77

0.25

0.68

1.69

0.06

0.09

0.18

0.19

0.00

0.04

0.25

0.00

0.11

0.06

0.00

0.14

0.00

0.04

3.11

0.44

2.98

3.06

0.12

1.11

0.72

1.00

1.02

0.50

0.75

0.51

0.79

0.19

0.34

0.58

0.06

0.17

0.18

0.19

0.17

0.11

0.19

0.09

0.14

0.00

0.11

0.50

0.68

0.58

0.00

0.09

0.04

0.00

0.26

0.11 


\section{ANEXO 1 (Continuación)}

Listado taxonómico de las mariposas y abundancia relativa (\%) registradas en las cercas vivas multiestrato (CVM) y simples (CVS), en Esparza, Costa Rica. 2005- 2006.

ANNEX 1 (Continued)

Butterfly taxonomic list and relative abundance (\%) in multi-strata live fences (CVM) and simple live fences (CVS) observed in Esparza, Costa Rica. 2005-2006

\begin{tabular}{|c|c|c|c|}
\hline \multirow{2}{*}{ Especies } & \multicolumn{2}{|c|}{ Habitat } & \multirow{2}{*}{ Total } \\
\hline & CVM & CVS & \\
\hline \multicolumn{4}{|l|}{ Subfamilia Biblinae } \\
\hline Callicore phiteas Latreille, 1811 & 0.00 & 0.09 & 0.04 \\
\hline Eunica mygdonia Godart, 1824 & 0.31 & 1.62 & 0.86 \\
\hline Hamadryas februa ferentina Godart, 1824 & 3.36 & 1.79 & 2.70 \\
\hline Hamadryas guatemalena H. W. Bates, 1864 & 0.25 & 0.17 & 0.22 \\
\hline Nica flavilla canthara Doubleday, 1849 & 0.00 & 0.09 & 0.04 \\
\hline \multicolumn{4}{|l|}{ Subfamilia Nymphalidae } \\
\hline Anartia fatima Godart, 1824 & 8.03 & 6.81 & 7.51 \\
\hline Anartia jatrophae Linnaeus, 1763 & 0.50 & 0.26 & 0.40 \\
\hline Castilia griseobasalis Röbert, 1913 & 0.12 & 0.00 & 0.07 \\
\hline Castilia ofella Hewitson, 1854 & 0.06 & 0.00 & 0.04 \\
\hline Chlosyne hippodrome Geyer, 1837 & 0.37 & 0.26 & 0.32 \\
\hline Chlosyne lacinia Geyer, 1837 & 0.12 & 0.60 & 0.32 \\
\hline Colobura dirce dirce Linnaeus, 1758 & 0.06 & 0.09 & 0.07 \\
\hline Junonia evarete Cramer, 1775 & 4.73 & 9.87 & 6.90 \\
\hline Microtia elva H. W. Bates, 1867 & 0.87 & 0.17 & 0.58 \\
\hline Siproeta epaphus Latreille, 1819 & 0.06 & 0.00 & 0.04 \\
\hline Siproeta stelenes Linnaeus, 1758 & 2.74 & 2.47 & 2.62 \\
\hline Smyrna blomfilda datis Fruhstofer, 1908 & 0.19 & 0.09 & 0.14 \\
\hline Thessalia theona Ménétriés, 1855 & 0.12 & 0.00 & 0.07 \\
\hline No. de individuos totales & 1605 & 1177 & 2782 \\
\hline No. de especies & 70 & 51 & 75 \\
\hline
\end{tabular}


